\title{
Axion Search with a Quantum-Limited Ferromagnetic Haloscope
}

\author{
N. Crescini $\odot,{ }^{1,2, *}$ D. Alesini, ${ }^{3}$ C. Braggio $\odot,{ }^{2,4}$ G. Carugno, ${ }^{2,4}$ D. D’Agostino $\odot,{ }^{5}$ D. Di Gioacchino $\odot,{ }^{3}$ \\ P. Falferi, ${ }^{6}$ U. Gambardella $\odot,{ }^{5}$ C. Gatti $\odot,{ }^{3}$ G. Iannone, ${ }^{5}$ C. Ligi $\odot,{ }^{3}$ A. Lombardi $\odot,{ }^{1}$ A. Ortolan $\odot,{ }^{1}$ \\ R. Pengo $\odot,{ }^{1}$ G. Ruoso $\odot,{ }^{1, \dagger}$ and L. Taffarello ${ }^{4}$
}

(QUAX Collaboration)

\author{
${ }^{1}$ INFN-Laboratori Nazionali di Legnaro, Viale dell'Università 2, 35020 Legnaro (PD), Italy \\ ${ }^{2}$ Dipartimento di Fisica e Astronomia, Via Marzolo 8, 35131 Padova, Italy \\ ${ }^{3}$ INFN-Laboratori Nazionali di Frascati, Via Enrico Fermi 40, 00044 Roma, Italy \\ ${ }^{4}$ INFN-Sezione di Padova, Via Marzolo 8, 35131 Padova, Italy \\ ${ }^{5}$ INFN-Sezione di Napoli, Via Cinthia, 80126 Napoli, Italy and Dipartimento di Fisica, \\ Via Giovanni Paolo II 132, 84084 Fisciano (SA), Italy \\ ${ }^{6}$ IFN-CNR, Fondazione Bruno Kessler, and INFN-TIFPA, Via alla Cascata 56, 38123 Povo (TN), Italy
}

(Received 24 January 2020; revised manuscript received 12 March 2020; accepted 17 April 2020; published 1 May 2020)

\begin{abstract}
A ferromagnetic axion haloscope searches for dark matter in the form of axions by exploiting their interaction with electronic spins. It is composed of an axion-to-electromagnetic field transducer coupled to a sensitive rf detector. The former is a photon-magnon hybrid system, and the latter is based on a quantum-limited Josephson parametric amplifier. The hybrid system consists of ten $2.1 \mathrm{~mm}$ diameter yttrium iron garnet spheres coupled to a single microwave cavity mode by means of a static magnetic field. Our setup is the most sensitive rf spin magnetometer ever realized. The minimum detectable field is $5.5 \times 10^{-19} \mathrm{~T}$ with $9 \mathrm{~h}$ integration time, corresponding to a limit on the axion-electron coupling constant $g_{a e e} \leq 1.7 \times 10^{-11}$ at $95 \%$ C.L. The scientific run of our haloscope resulted in the best limit on dark matter axions to electron coupling constant in a frequency span of about $120 \mathrm{MHz}$, corresponding to the axion-mass range 42.4-43.1 $\mu \mathrm{eV}$. This is also the first apparatus to perform a wide axion-mass scanning by only changing the static magnetic field.
\end{abstract}

DOI: 10.1103/PhysRevLett.124.171801

The axion is a beyond the standard model (BSM) hypothetical particle, first introduced in the 1970s as a consequence of the strong $C P$ problem of quantum chromodynamics (QCD) [1-3]. Present experimental efforts are directed toward "invisible" axions, described by the KimShifman-Vainshtein-Zakharov [4,5] and Dine-FischlerSrednicki-Zhitnitsky (DFSZ) [6,7] models, which are extremely light and weakly coupled to the standard model particles. Axions can be produced in the early Universe by different mechanisms [8-12] and may be the main constituents of galactic dark matter (DM) halos. Astrophysical and cosmological constraints [13,14], as well as lattice QCD calculations of the DM density [15-17], provide a preferred axion-mass window around tens of $\mu \mathrm{eV}$.

Nonbaryonic DM is where cosmology meets particle physics, and axions are among the most interesting and

Published by the American Physical Society under the terms of the Creative Commons Attribution 4.0 International license. Further distribution of this work must maintain attribution to the author(s) and the published article's title, journal citation, and DOI. Funded by SCOAP ${ }^{3}$. challenging BSM particles to detect. Their experimental search can be carried out with Earth-based instruments immersed in the Milky Way's halo, which are therefore called "haloscopes" [18]. Nowadays, haloscopes rely on the inverse Primakoff effect to detect axion-induced excess photons inside a microwave cavity in a static magnetic field. Primakoff haloscopes allowed us to exclude axions with masses $m_{a}$ between 1.91 and $3.69 \mu \mathrm{eV}$ [19-21] and, together with helioscopes [22], are the only experiments that reached the QCD-axion parameter space. The last years saw a flourishing of new ideas to search for axions and axionlike particles (ALPs) [23-35]. Among these, the QUAX experiment $[36,37]$ searches for DM axions through their coupling with the spin of the electron. This experiment aims to implement the idea of Ref. [38] as follows.

The axion-electron interaction is described by the Lagrangian

$$
\mathcal{L}_{a e}=\frac{g_{a e e}}{2 m_{e}} \partial_{\mu} a\left(\bar{\psi}_{e} \gamma^{\mu} \gamma_{5} \psi_{e}\right),
$$

where $g_{a e e}$ is the axion-electron coupling constant, $a$ is the axion field, $\psi_{e}$ and $m_{e}$ are the electron wave function and 
mass, and $\gamma_{\mu}$ and $\gamma_{5}$ are Dirac matrices. This vertex describes an axion-induced flip of an electron spin, which then decays back to the ground state emitting a photon. Since $v_{a}$, the relative speed between Earth and the DM halo, is small, we may use the nonrelativistic limit of EulerLagrange equations and recast the interaction term

$$
\mathcal{L}_{a e} \simeq-2 \mu_{B} \boldsymbol{\sigma} \cdot\left(\frac{g_{a e e}}{2 e}\right) \nabla a \equiv-2 \mu_{B} \boldsymbol{\sigma} \cdot \mathbf{B}_{a} .
$$

Here $-2 \mu_{B} \sigma$ and $e$ are the spin and charge of the electron, $\mu_{B}$ is the Bohr magneton, and $\mathbf{B}_{a}$ is defined as the axion effective magnetic field. As $\nabla a \propto v_{a}$ [38], the nonzero value of $v_{a}$ results in $\mathbf{B}_{a} \neq 0$.

If accounting for the whole DM, the numeric axion density is $n_{a} \simeq 8 \times 10^{12}\left(42 \mu \mathrm{eV} / m_{a}\right) \mathrm{cm}^{-3}$. For $v_{a} \simeq 10^{-3} c$, where $c$ is the speed of light, the de Broglie wavelength and coherence time of the galactic axion field are $\lambda_{\nabla a}=25\left(42 \mu \mathrm{eV} / m_{a}\right) \mathrm{m}$, and $\tau_{\nabla a}=55\left(m_{a} / 42 \mu \mathrm{eV}\right) \mu \mathrm{s}$ [36,37]. The effective field frequency is proportional to the axion mass, $\omega_{a} / 2 \pi=10\left(m_{a} / 42 \mu \mathrm{eV}\right) \mathrm{GHz}$, while its amplitude depends on the properties of the DM halo and of the axion model,

$$
B_{a}=\frac{g_{a e e}}{2 e} \sqrt{\frac{n_{a} \hbar}{m_{a} c}} m_{a} v_{a} \simeq 4 \times 10^{-23}\left(\frac{m_{a}}{42 \mu \mathrm{eV}}\right) \mathrm{T},
$$

where $\hbar$ is the reduced Planck constant. These features allow for the driving of a coherent interaction between $\mathbf{B}_{a}$ and the homogeneous magnetization of a macroscopic sample. The sample is immersed in a static magnetic field $B_{0}$ to couple the axion field to the Kittel mode of uniform precession of the magnetization. The interaction yields a conversion rate of axions to magnons, which can be measured by searching for oscillations in the sample's magnetization. Because of the angle between $\mathbf{B}_{0}$ and $\mathbf{B}_{a}$, the resulting signal undergoes a full daily modulation [39]. The maximum axion-deposited power is related to Eq. (3) and to the characteristics of the receiver, namely, number of spins $N_{s}$ and system relaxation time $\tau_{s}$

$$
P_{a}=\gamma_{e} \mu_{B} N_{s} \omega_{a} B_{a}^{2} \tau_{s},
$$

where $\gamma_{e}$ is the electron gyromagnetic ratio.

To detect this signal, we devised a suitable receiver. As it measures the magnetization of a sample, it is configured as a spin magnetometer used as an axion haloscope. The device consists of an axion field transducer and of a $\mathrm{rf}$ detection chain.

At high frequencies and in free field, the electron spin resonance linewidth is dominated by radiation damping, which limits $\tau_{s}$ [40-42]. To avoid this issue, the material is placed in a microwave cavity. If the frequency of the Kittel mode $\omega_{m}=\gamma_{e} B_{0}$ is close to the cavity mode frequency $\omega_{c}$, the two resonances hybridize and the single mode splits into two, following an anticrossing curve $[43,44]$.
The $B_{0}$-dependent hybrid modes' frequencies are $\omega_{1}$ and $\omega_{2}$ and the cavity-material coupling is $g_{\mathrm{cm}}=$ $\min \left(\omega_{2}-\omega_{1}\right)$. If $g_{c m}$ is larger than the hybrid mode linewidths $\gamma_{1,2} \simeq\left(\gamma_{c}+\gamma_{m}\right) / 2$, where $\gamma_{c}$ and $\gamma_{m}$ are the cavity and material dissipations, the system is in the strong coupling regime. To increase $P_{a}, N_{s}$ and $\tau_{s}$ must be large, so a suitable sample has a high spin density and a narrow linewidth. The best material identified so far is yttrium iron garnet (YIG), with roughly $2 \times 10^{22} \mathrm{spins} / \mathrm{cm}^{3}$ and $1 \mathrm{MHz}$ linewidth [45].

In the apparatus that we operated at the Laboratori Nazionali di Legnaro of INFN, the TM110 mode of a cylindrical copper cavity is coupled to ten $2.1 \mathrm{~mm}$ diameter spheres of YIG. The spherical shape is needed to avoid geometrical demagnetization. We devised an on-site grinding and polishing procedure to obtain narrow linewidth spherical samples starting from large single crystals of YIG. The spheres are placed on the axis of the cavity, where the rf magnetic field is uniform.

Several room temperature tests were performed to design the YIG holder: a $4 \mathrm{~mm}$ inner diameter fused silica pipe, containing ten stacked PTFE cups, each one large enough to host a free rotating YIG. Free rotation permits the spheres' easy axis self-alignment to the external magnetic field, while a separation of $3 \mathrm{~mm}$ prevents sphere-sphere interaction. The pipe is filled with 1 bar of helium and anchored to the cavity for thermalization. The cavity and pipe are placed inside the internal vacuum chamber (IVC) of a dilution refrigerator, with a base temperature around $90 \mathrm{mK}$. Outside the IVC, in a liquid helium bath, a superconducting magnet provides the static field with an inhomogeneity below $7 \mathrm{ppm}$ over all the spheres.

The resulting hybrid system (HS) has been studied by collecting a $B_{0}$ vs frequency transmission plot, reported in Fig. 1 (left). The measured plot is not a usual anticrossing curve. In our system, the cavity frequency $\omega_{c} / 2 \pi=$ $10.7 \mathrm{GHz}$ and the expected coupling is of the order of $600 \mathrm{MHz}$, thus $\omega_{2}$ gets close and couples to a higher order mode of the cavity. This hybrid mode further splits into others, making the two oscillators description unsuitable. Other disturbances are related to residual sphere-sphere interaction and to nonidentical spheres. To model the HS, we write a Hamiltonian based on two cavity modes, $c$ and $d$, and two magnetic modes, $m$ and $n$,

$$
\mathcal{H}_{c d m n}=\left(\begin{array}{cccc}
\omega_{c}-\frac{i \gamma_{c}}{2} & 0 & g_{c m} & g_{c n} \\
0 & \omega_{d}-\frac{i \gamma_{d}}{2} & g_{d m} & g_{d n} \\
g_{c m} & g_{d m} & \omega_{m}-\frac{i \gamma_{m}}{2} & g_{m n} \\
g_{c n} & g_{d n} & g_{m n} & \omega_{n}-\frac{i \gamma_{n}}{2}
\end{array}\right),
$$

where $g, \omega$, and $\gamma$ indicate their couplings, resonant frequencies, and dissipations, respectively. Figure 1 (right) shows the function $f_{c d m n}\left(\omega, \omega_{m}\right)=\operatorname{det}\left(\omega \mathbb{I}_{4}-\mathcal{H}_{c d m n}\right)$, whose maxima identify the resonance frequencies of the 


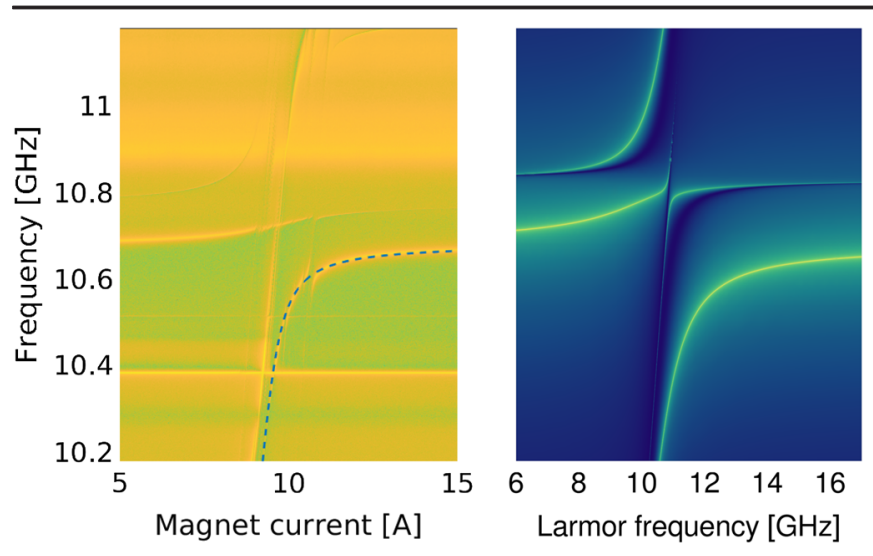

FIG. 1. Measured (left) and modeled (right) transmission functions of the HS. The right plot is the function $f_{c d m n}\left(\omega, \omega_{m}\right)$, based on the second quantization of coupled harmonic oscillators, while the left one is a SO-to-readout (see Fig. 2) transmission measurement with the JPA off, performed at $90 \mathrm{mK}$. Color scales are in arbitrary units (brighter colors correspond to higher amplitudes). The dashed line in the left plot identifies the low-frequency hybrid mode, having resonant frequencies $\omega_{1}$ and linewidths $\gamma_{1}$.

HS. By comparing the two plots of Fig. 1, one can see that the model appropriately describes the system, allowing us to extract the linewidths, frequencies, and couplings of the modes through a fit. The typical measured values are $\gamma_{1} \simeq$ $1.9 \mathrm{MHz}$ and $g_{c m} \simeq 638 \mathrm{MHz}$, yielding $\tau_{s} \simeq 84 \mathrm{~ns}$ and $N_{s} \simeq$ $1.0 \times 10^{21}$ spins, respectively. With respect to the hybridization of a single sphere, $g_{\mathrm{cm}}$ scales as the square root of the number of spheres, showing their coherent coupling to the cavity mode. Remarkably, the mode $\omega_{1}$ is not altered by other modes, thus we will use it to search for axion-induced signals. For a fixed $B_{0}$ the linewidth of the hybrid mode is the haloscope sensitive band. By changing $B_{0}$, we can perform a frequency scan along the dashed line of Fig. 1.

The electronic schematics, shown in Fig. 2, consists of four rf lines used to characterize, calibrate, and operate the haloscope. The HS output power is collected by a dipole antenna $(D 1)$, connected to a manipulator by a thin steel wire and a system of pulleys to change its coupling. The source oscillator ( $\mathrm{SO}$ ) line is connected to a weakly coupled antenna (D2) and used to inject signals into the HS, the pump line goes to a Josephson parametric amplifier (JPA), the readout line amplifies the power collected by $D 1$, and "Aux" is an auxiliary line. The readout line is connected to a heterodyne as described in [37], where an analogto-digital converter (ADC) samples the down-converted power, which is then stored for analysis. The JPA is a quantum-limited amplifier, with resonance frequency of about $10 \mathrm{GHz}$, resulting in a noise temperature of $0.5 \mathrm{~K}$. Its gain is close to $20 \mathrm{~dB}$ in a band of order $10 \mathrm{MHz}$, and its working frequency can be tuned thanks to a small superconducting coil [46]. Excluding some mode crossings, hybrid mode and JPA frequencies overlap between 10.2 and $10.4 \mathrm{GHz}$ and allow us to scan the corresponding axion-mass range.

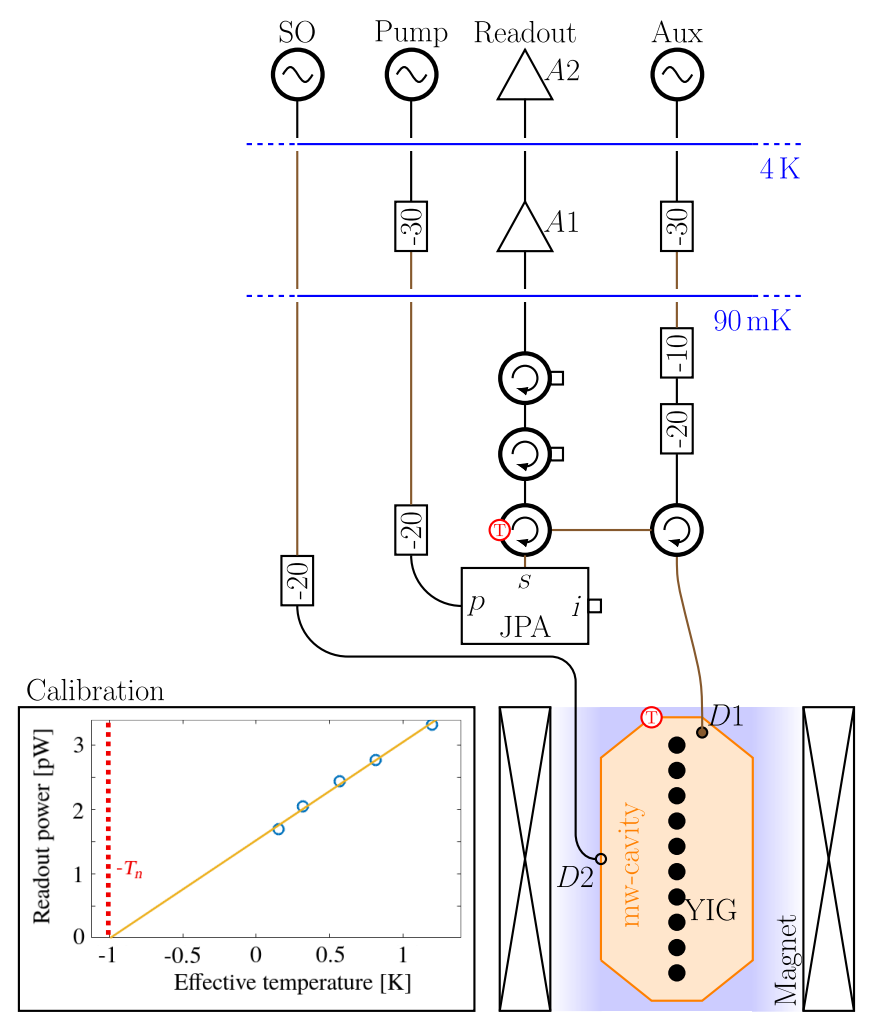

FIG. 2. Schematics of the apparatus. The cavity is reported in orange, the ten YIG spheres are in black, and the blue shaded region is permeated by a uniform magnetic field. The cryogenic and room temperature HEMT amplifiers are $A 1$ and $A 2$, respectively, and the JPA ports are the signal $(s)$, idler $(i)$, and pump $(p)$. Superconducting cables are brown, the red circled $T$ 's are the thermometers, SO is a source oscillator, and attenuators are shown with their reduction factor in decibels. (Inset) The calibration of the system gain and noise temperature, obtained by injecting signals in the SO line. The power injected in the HS is given in terms of an effective temperature proportional to $A_{\text {cal }}$. The errors are within the symbol dimension. See text for further details.

The procedure to calibrate all the lines of the setup is as follows: (i) the transmittivity of the Aux-readout path $K_{\mathrm{AR}}$ is measured by decoupling $D 1$ or by detuning $\omega_{1}$; (ii) for the Aux-SO and SO-readout paths, $K_{\mathrm{AS}}$ and $K_{\mathrm{SR}}$ are obtained by critically coupling $D 1$ to the mode $\omega_{1}$. The transmittivity of the $\mathrm{SO}$ line is $K_{\mathrm{SO}} \simeq \sqrt{K_{\mathrm{SR}} K_{\mathrm{AS}} / K_{\mathrm{AR}}}$. If a signal of power $A_{\text {in }}$ is injected in the SO line, the fraction of this power getting into the HS results $A_{\text {cal }}=A_{\text {in }} K_{\text {SO }}$. Since $A_{\text {cal }}$ is a calibrated signal, it can be used to measure gain and noise temperature of the readout line. From this measurement, we obtain a system noise temperature $T_{n}=1.0 \mathrm{~K}$ and a gain of $70.4 \mathrm{~dB}$ from $D 1$ to readout (see Fig. 2). In our setup, the coupling of $D 1$ can be varied by $8 \mathrm{~dB}$, thus we estimate a calibration uncertainty of $16 \%$.

To double check the accuracy of the result, we measure the thermal noise of the HS. The noise difference for $\omega_{1}$ on and off the JPA resonance (dark blue and light blue) gives 


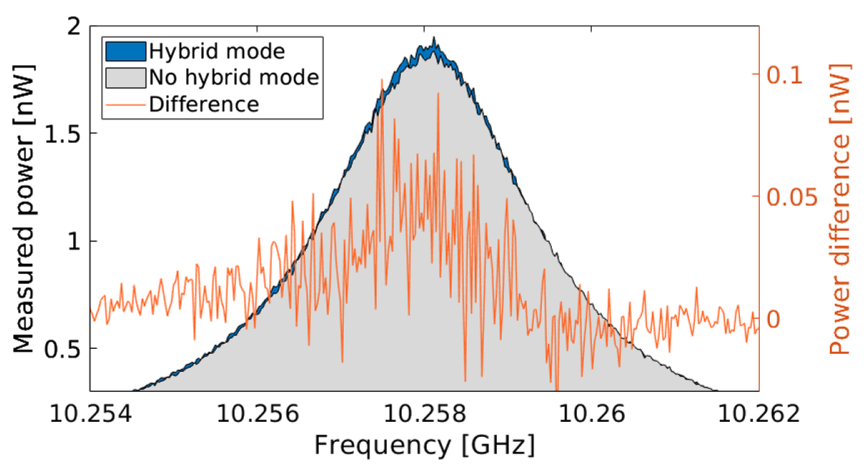

FIG. 3. Excess noise in the JPA band due to the added thermal noise of the HS. The areas show the power measured at the readout with $\omega_{1}$ on resonance with the JPA (blue) and detuned from the JPA bandwidth (gray). The difference of the two is the HS noise (reported in orange).

the noise added by the hybrid mode (orange curve), as shown in Fig. 3. The excess noise is compatible with a temperature of the HS $\sim 100 \mathrm{mK}$ higher than the one of the nearest load, which is realistic since the load is closer to the mixing chamber at $90 \mathrm{mK}$. Similar results are obtained by changing the $D 1$ antenna coupling for a fixed $B_{0}$. We measured the JPA gain, the HEMT's noise temperature, and estimate the HS temperature to get the noise budget detailed in Table I. The $0.14 \mathrm{~K}$ difference may be due to unaccounted losses or nonprecise temperature control.

As $\lambda_{\nabla a}$ is much larger than the scale of the apparatus, the axion field interacts coherently with all the spheres. Its quality factor is $\omega_{a} \tau_{\nabla a} / 2 \simeq 2 \times 10^{6}$, thus the axion power is deposited in a band of about $5 \mathrm{kHz}$.

The axion search consisted of 56 runs, each one with fixed $B_{0}$. For every run, a transmission measurement of the hybrid system is used to set $\omega_{1}$, to critically couple $D 1$ to it, and to measure $\gamma_{1}$. The frequency stability of $\omega_{1}$ resulted well below the linewidth within an interval of several hours, allowing long integration times. Data are stored with the ADC over a $2 \mathrm{MHz}$ band around $\omega_{1}$ for subsequent analysis. We FFT the data with a $100 \mathrm{~Hz}$ resolution bandwidth to identify and remove biased bins and disturbances in the down-converted spectra. To estimate the sensitivity to the axion field, we rebin the FFTs with a resolution bandwidth $\mathrm{RBW} \simeq 5 \mathrm{kHz}$, which at this frequency gives the best SNR for the axionic signal [38].

TABLE I. Noise budget of the apparatus, expressed as equivalent noise temperature. The measured noise is compatible with the estimated one.

\begin{tabular}{lc}
\hline \hline Source & Noise $[\mathrm{K}]$ \\
\hline Quantum noise & 0.50 \\
Thermal noise & 0.10 \\
HEMT's noise & 0.25 \\
Expected total & 0.85 \\
Measured total $T_{n}$ & 0.99 \\
\hline \hline
\end{tabular}

The spectra are fitted to a degree five polynomial to extract the residuals, whose standard deviation is the sensitivity of the apparatus. We verified that the analysis procedure excludes unwanted bins, while preserving the signal and SNR by adding a fake axion signal to the acquired data.

Our data were collected in July 2019 in a total run time of $74 \mathrm{~h}$. The average run length was $\sim 1 \mathrm{~h}$, and each one was performed during the maximum of the daily-modulated axionic signal. The measured fluctuations are compatible with the estimated noise in every run, and we detected no statistically significant signal consistent with the DM axion field. The minimum measured fluctuation is $\sigma_{P}=$ $5.1 \times 10^{-24} \mathrm{~W}$, for the longest integration time $t=9 \mathrm{~h}$, where the Dicke prediction is $k_{B} T_{n} \sqrt{\mathrm{RBW} / t}=$ $4.8 \times 10^{-24} \mathrm{~W}$. By using Eq. (4), one obtains the magnetic field sensitivity $\sigma_{B}=5.5 \times 10^{-19} \mathrm{~T}$, which, to our knowledge, is a record one for a rf spin magnetometer. The absence of fast rf bursts in the data is verified by using a $1 \mathrm{~ms}$ time resolution waterfall spectrogram.

Even if the minimum field detectable by the haloscope is much larger than $B_{a}$, these measurements can still be a probe for ALPs, which may also constitute the totality of DM [47]. The 95\% C.L. upper limit on the axion-electron coupling constant is

$$
g_{a e e}<\frac{e}{\pi m_{a} v_{a}} \sqrt{\frac{k_{a c} \times 2 \sigma_{P}}{2 \mu_{B} \gamma_{e} n_{a} N_{s} \tau_{s}}} \simeq 1.7 \times 10^{-11} .
$$

The transduction coefficient of the axionic signal $k_{a c}$ was calculated with a model similar to the one of Eq. (5) [48]. It essentially depends on $\omega_{1}$ and, in our bandwidth, results $0.5<k_{a c}<1.0$. The overall exclusion plot obtained with the ferromagnetic haloscope is given in Fig. 4. All the experimental parameters used to extract the limits from Eq. (6) are measured within every run, making the measurement highly self-consistent.

These results improved the best previous limits [37] by roughly a factor 30 in $g_{a e e}$ and 50 in bandwidth. The improvement over the previous prototype is due to an increased material volume, to an almost quantum-limited noise temperature, and to longer integration times. Much narrower axion-mass scans were performed by previous experiments of this kind [37,49], and we now demonstrate that it is feasible to tune a hybrid resonance over hundreds of megahertz to search for axion-deposited power. Our prototype scanned a range of axion masses of about $0.7 \mu \mathrm{eV}$ with a field variation of $7 \mathrm{mT}$, drastically simplifying the tuning of the haloscope.

In conclusion, we designed and developed a quantumlimited rf spin magnetometer used as an axion haloscope. The instrument implements an axion-to-rf transducer, i.e., a hybrid system that embeds one of the largest quantities of magnetic material to date, and a detection electronics based on a quantum-limited JPA. The operation of this instrument led to an axion search over a span of $0.7 \mu \mathrm{eV}$ around $42.7 \mu \mathrm{eV}$, 


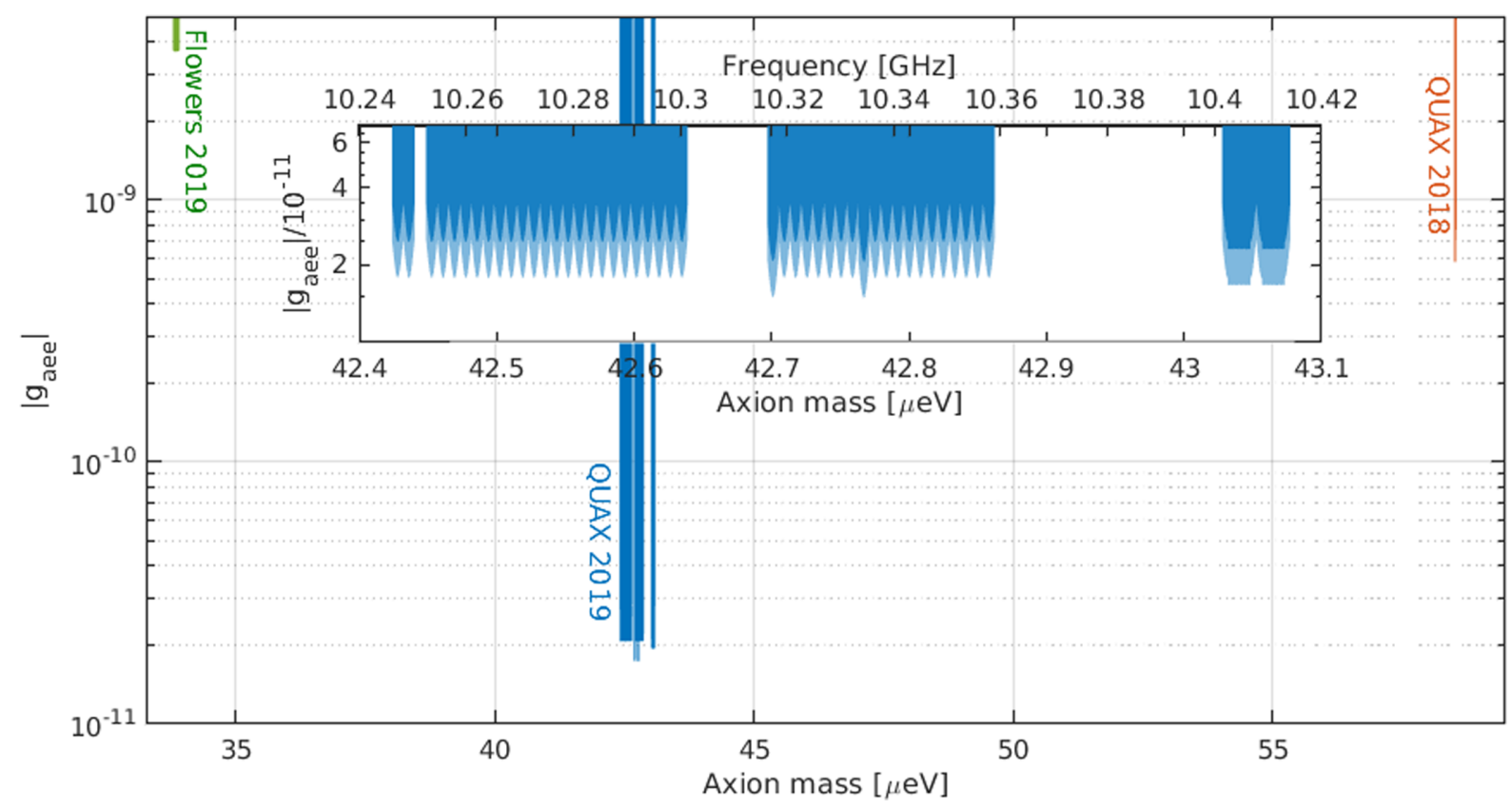

FIG. 4. Exclusion plot at $95 \%$ C.L. on the axion-electron coupling obtained with the present prototype (excluded region reported in blue and error in light blue) and overview of other searches for the axion-electron interaction. The other results are from [37] (orange) and [49] (green), while the DFSZ axion line is at about $g_{a e e} \simeq 10^{-15}$. (Inset) A detailed view of the reported result.

with a maximum sensitivity to $g_{a e e}$ of $1.7 \times 10^{-11}$. This, to our knowledge, is the best reported limit on the coupling of DM axions to electrons, and corresponds to a $1-\sigma$ field sensitivity of $5.5 \times 10^{-19} \mathrm{~T}$, which is a record one. No show stoppers have been found so far, and hence a further upscale of the system can be foreseen. A superconducting cavity with a higher quality factor was already developed and tested [50]. It was not employed in this Letter since the YIG linewidth does not match the superconducting cavity one, and the improvement on the setup would have been negligible. With this prototype, we reached the rf sensitivity limit of linear amplifiers [51]. To further improve the present setup, one needs to rely on bolometers or single magnon or photon counter [52]. Such devices are currently being studied by a number of groups, as they find important applications in the field of quantum information [53-56].

We are grateful to E. Berto, A. Benato, and M. Rebeschini, who did the mechanical work, F. Calaon and M. Tessaro who helped with the electronics and cryogenics, and to F. Stivanello for the chemical treatments. We thank G. Galet and L. Castellani for the development of the magnet power supply and M. Zago who realized the technical drawings of the system. We deeply acknowledge the Cryogenic Service of the Laboratori Nazionali di Legnaro for providing us large quantities of liquid helium on demand.

*nicolo.crescini@phd.unipd.it

†ruoso@lnl.infn.it
[1] R. D. Peccei and H.R. Quinn, $C P$ Conservation in the Presence of Pseudoparticles, Phys. Rev. Lett. 38, 1440 (1977).

[2] S. Weinberg, A New Light Boson?, Phys. Rev. Lett. 40, 223 (1978).

[3] F. Wilczek, Problem of Strong $p$ and $t$ Invariance in the Presence of Instantons, Phys. Rev. Lett. 40, 279 (1978).

[4] J. E. Kim, Weak-Interaction Singlet and Strong CP Invariance, Phys. Rev. Lett. 43, 103 (1979).

[5] M. A. Shifman, A. I. Vainshtein, and V. I. Zakharov, Can confinement ensure natural $C P$ invariance of strong interactions?, Nucl. Phys. B166, 493 (1980).

[6] M. Dine, W. Fischler, and M. Srednicki, A simple solution to the strong $C P$ problem with a harmless axion, Phys. Lett. 104B, 199 (1981).

[7] A. R. Zhitnitsky, The Weinberg model of the $C P$ violation and $t$ odd correlations in weak decays, Yad. Fiz. 31, 1024 (1980) [Sov. J. Nucl. Phys. 31, 529 (1980)].

[8] M. Dine and W. Fischler, The not-so-harmless axion, Phys. Lett. 120B, 137 (1983).

[9] J. Preskill, M. B. Wise, and F. Wilczek, Cosmology of the invisible axion, Phys. Lett. 120B, 127 (1983).

[10] P. Sikivie, in Axion Cosmology (Springer, Berlin, Heidelberg, 2008), pp. 19-50.

[11] L. D. Duffy and K. van Bibber, Axions as dark matter particles, New J. Phys. 11, 105008 (2009).

[12] D. J. E. Marsh, Axion cosmology, Phys. Rep. 643, 1 (2016).

[13] G. G. Raffelt, Astrophysical methods to constrain axions and other novel particle phenomena, Phys. Rep. 198, 1 (1990).

[14] M. S. Turner, Windows on the axion, Phys. Rep. 197, 67 (1990).

[15] S. Borsányi, Z. Fodor, J. Guenther, K.-H. Kampert, S. D. Katz, T. Kawanai, T. G. Kovacs, S. W. Mages, A. Pasztor, 
F. Pittler et al., Calculation of the axion mass based on high-temperature lattice quantum chromodynamics, Nature (London) 539, 69 (2016).

[16] E. Berkowitz, M. I. Buchoff, and E. Rinaldi, Lattice QCD input for axion cosmology, Phys. Rev. D 92, 034507 (2015).

[17] L. Di Luzio, F. Mescia, and E. Nardi, Redefining the Axion Window, Phys. Rev. Lett. 118, 031801 (2017).

[18] P. Sikivie, Experimental Tests of the "Invisible" Axion, Phys. Rev. Lett. 51, 1415 (1983).

[19] N. Du, N. Force, R. Khatiwada, E. Lentz, R. Ottens, L. J. Rosenberg, G. Rybka, G. Carosi, N. Woollett, D. Bowring, A. S. Chou, A. Sonnenschein, W. Wester, C. Boutan et al., Search for Invisible Axion Dark Matter with the Axion Dark Matter Experiment, Phys. Rev. Lett. 120, 151301 (2018).

[20] S. J. Asztalos, G. Carosi, C. Hagmann, D. Kinion, K. van Bibber, M. Hotz, L. J Rosenberg, G. Rybka, J. Hoskins, J. Hwang, P. Sikivie, D. B. Tanner, R. Bradley, and J. Clarke, Squid-Based Microwave Cavity Search for Dark-Matter Axions, Phys. Rev. Lett. 104, 041301 (2010).

[21] T. Braine, R. Cervantes, N. Crisosto, N. Du, S. Kimes, L. J Rosenberg, G. Rybka, J. Yang, D. Bowring, A. S. Chou, R. Khatiwada, A. Sonnenschein, W. Wester, G. Carosi et al., Extended search for the invisible axion with the axion dark matter experiment, Phys. Rev. Lett. 124, 101303 (2020).

[22] V. Anastassopoulos, S. Aune, K. Barth, A. Belov, H. Bräuninger, G. Cantatore, J. M. Carmona, J. F. Castel, S. A. Cetin, F. Christensen, J. I. Collar, T. Dafni, M. Davenport, T. A. Decker et al., New cast limit on the axion-photon interaction, Nat. Phys. 13, 584 (2017).

[23] P. W. Graham, I. G. Irastorza, S. K. Lamoreaux, A. Lindner, and K. A. van Bibber, Experimental searches for the axion and axion-like particles, Annu. Rev. Nucl. Part. Sci. 65, 485 (2015).

[24] J. Jaeckel and A. Ringwald, The low-energy frontier of particle physics, Annu. Rev. Nucl. Part. Sci. 60, 405 (2010).

[25] I. G. Irastorza and J. Redondo, New experimental approaches in the search for axion-like particles, Prog. Part. Nucl. Phys. 102, 89 (2018).

[26] A. Caldwell, G. Dvali, B. Majorovits, A. Millar, G. Raffelt, J. Redondo, O. Reimann, F. Simon, F. Steffen, and MADMAX Working Group et al., Dielectric Haloscopes: A New Way to Detect Axion Dark Matter, Phys. Rev. Lett. 118, 091801 (2017).

[27] D. Budker, P. W. Graham, M. Ledbetter, S. Rajendran, and A. O. Sushkov, Proposal for a Cosmic Axion Spin Precession Experiment (CASPEr), Phys. Rev. X 4, 021030 (2014).

[28] B. T. McAllister, G. Flower, E. N. Ivanov, M. Goryachev, J. Bourhill, and M.E. Tobar, The ORGAN experiment: An axion haloscope above $15 \mathrm{GHz}$, Phys. Dark Universe 18, 67 (2017).

[29] J. L. Ouellet, C. P. Salemi, J. W. Foster, R. Henning, Z. Bogorad, J. M. Conrad et al., First Results from ABRACADABRA-10 cm: A Search for Sub- $\mu \mathrm{eV}$ Axion Dark Matter, Phys. Rev. Lett. 122, 121802 (2019).

[30] R. Ballou, G. Deferne, M. Finger, M. Finger, L. Flekova, J. Hosek, S. Kunc, K. Macuchova, K. A. Meissner, P. Pugnat, M. Schott, A. Siemko, M. Slunecka, M. Sulc, C. Weinsheimer, and J. Zicha, New exclusion limits on scalar and pseudoscalar axionlike particles from light shining through a wall, Phys. Rev. D 92, 092002 (2015).

[31] K. Ehret, M. Frede, S. Ghazaryan, M. Hildebrandt, E.-A. Knabbe, D. Kracht, A. Lindner, J. List, T. Meier, N. Meyer, D. Notz, J. Redondo, A. Ringwald, G. Wiedemann, and B. Willke, New alps results on hidden-sector lightweights, Phys. Lett. B 689, 149 (2010).

[32] T. Wu, J. W. Blanchard, G. P. Centers, N. L. Figueroa, A. Garcon, P. W. Graham, D. F. Jackson Kimball, S. Rajendran, Y. V. Stadnik, A. O. Sushkov, A. Wickenbrock, and D. Budker, Search for Axionlike Dark Matter with a LiquidState Nuclear Spin Comagnetometer, Phys. Rev. Lett. 122, 191302 (2019).

[33] J. Lee, A. Almasi, and M. Romalis, Improved Limits on Spin-Mass Interactions, Phys. Rev. Lett. 120, 161801 (2018).

[34] N. Crescini, C. Braggio, G. Carugno, P. Falferi, A. Ortolan, and G. Ruoso, Improved constraints on monopole-dipole interaction mediated by pseudo-scalar bosons, Phys. Lett. B 773, 677 (2017).

[35] M. Tanabashi et al., Review of particle physics, Phys. Rev. D 98, 030001 (2018).

[36] R. Barbieri, C. Braggio, G. Carugno, C. S. Gallo, A. Lombardi, A. Ortolan, R. Pengo, G. Ruoso, and C. C. Speake, Searching for galactic axions through magnetized media: The QUAX proposal, Phys. Dark Universe 15, 135 (2017).

[37] N. Crescini, D. Alesini, C. Braggio, G. Carugno, D. Di Gioacchino, C. S. Gallo, U. Gambardella, C. Gatti, G. Iannone, G. Lamanna, C. Ligi, A. Lombardi, A. Ortolan, S. Pagano, R. Pengo, G. Ruoso, C. C. Speake, and L. Taffarello, Operation of a ferromagnetic axion haloscope at $m_{a}=58 \mu \mathrm{eV}$, Eur. Phys. J. C 78, 703 (2018).

[38] R. Barbieri, M. Cerdonio, G. Fiorentini, and S. Vitale, Axion to magnon conversion. A scheme for the detection of galactic axions, Phys. Lett. B 226, 357 (1989).

[39] S. Knirck, A. J. Millar, C. A. J. O’Hare, J. Redondo, and F. D. Steffen, Directional axion detection, J. Cosmol. Astropart. Phys. 11 (2018) 051.

[40] N. Bloembergen and R. V. Pound, Radiation damping in magnetic resonance experiments, Phys. Rev. 95, 8 (1954).

[41] S. Bloom, Effects of radiation damping on spin dynamics, J. Appl. Phys. 28, 800 (1957).

[42] M. P. Augustine, Transient properties of radiation damping, Prog. Nucl. Magn. Reson. Spectrosc. 40, 111 (2002).

[43] Y. Tabuchi, S. Ishino, T. Ishikawa, R. Yamazaki, K. Usami, and Y. Nakamura, Hybridizing Ferromagnetic Magnons and Microwave Photons in the Quantum Limit, Phys. Rev. Lett. 113, 083603 (2014).

[44] X. Zhang, C.-L. Zou, L. Jiang, and H. X. Tang, Strongly Coupled Magnons and Cavity Microwave Photons, Phys. Rev. Lett. 113, 156401 (2014).

[45] R. C. LeCraw, E. G. Spencer, and C. S. Porter, Ferromagnetic resonance line width in yttrium iron garnet single crystals, Phys. Rev. 110, 1311 (1958).

[46] N. Roch, E. Flurin, F. Nguyen, P. Morfin, P. CampagneIbarcq, M. H. Devoret, and B. Huard, Widely Tunable, Nondegenerate Three-Wave Mixing Microwave Device Operating near the Quantum Limit, Phys. Rev. Lett. 108, 147701 (2012). 
[47] P. Arias, D. Cadamuro, M. Goodsell, J. Jaeckel, J. Redondo, and A. Ringwald, WISPy cold dark matter, J. Cosmol. Astropart. Phys. 06 (2012) 013.

[48] N. Crescini, Towards the development of the ferromagnetic axion haloscope, Ph.D. thesis, Padova University, 2019.

[49] G. Flower, J. Bourhill, M. Goryachev, and M. E. Tobar, Broadening frequency range of a ferromagnetic axion haloscope with strongly coupled cavity-magnon polaritons, Phys. Dark Universe 25, 100306 (2019).

[50] D. Alesini, C. Braggio, G. Carugno, N. Crescini, D. D’Agostino, D. Di Gioacchino, R. Di Vora, P. Falferi, S. Gallo, U. Gambardella, C. Gatti, G. Iannone et al., Galactic axions search with a superconducting resonant cavity, Phys. Rev. D 99, 101101(R) (2019).

[51] A. A. Clerk, M. H. Devoret, S. M. Girvin, F. Marquardt, and R. J. Schoelkopf, Introduction to quantum noise, measurement, and amplification, Rev. Mod. Phys. 82, 1155 (2010).

[52] S. K. Lamoreaux, K. A. van Bibber, K. W. Lehnert, and G. Carosi, Analysis of single-photon and linear amplifier detectors for microwave cavity dark matter axion searches, Phys. Rev. D 88, 035020 (2013).

[53] J. Govenius, R. E. Lake, K. Y. Tan, and M. Möttönen, Detection of Zeptojoule Microwave Pulses Using Electrothermal Feedback in Proximity-Induced Josephson Junctions, Phys. Rev. Lett. 117, 030802 (2016).

[54] K. Inomata, Z. Lin, K. Koshino, W. D. Oliver, J.-S. Tsai, T. Yamamoto, and Y. Nakamura, Single microwave-photon detector using an artificial $\lambda$-type three-level system, Nat. Commun. 7, 12303 (2016).

[55] L. S. Kuzmin, A. S. Sobolev, C. Gatti, D. Di Gioacchino, N. Crescini, A. Gordeeva, and E. Il'ichev, Single photon counter based on a Josephson junction at $14 \mathrm{GHz}$ for searching galactic axions, IEEE Trans. Appl. Supercond. 28, 1 (2018).

[56] D. Lachance-Quirion, S. P. Wolski, Y. Tabuchi, S. Kono, K. Usami, and Y. Nakamura, Entanglement-based single-shot detection of a single magnon with a superconducting qubit, Science 367, 425 (2020). 